MODELING, IDENTIFICATION AND CONTROL, 2004, VOL. 25, No. 3, 159-172

doi:10.4173/mic.2004.3.2

\title{
Nonlinear Observer Design for a Nonlinear Cable/String FEM Model using Contraction Theory
}

\author{
YILMAZ TÜRKYILMAZ* and OLAV EGELAND*† \\ Keywords: Cables, nonlinear models, finite element method, observers, nonlinear \\ analysis
}

\begin{abstract}
Contraction theory is a recently developed nonlinear analysis tool which may be useful for solving a variety of nonlinear control problems. In this paper, using Contraction theory, a nonlinear observer is designed for a general nonlinear cable/ string FEM (Finite Element Method) model. The cable model is presented in the form of partial differential equations (PDE). Galerkin's method is then applied to obtain a set of ordinary differential equations such that the cable model is approximated by a FEM model. Based on the FEM model, a nonlinear observer is designed to estimate the cable configuration. It is shown that the estimated configuration converges exponentially to the actual configuration. Numerical results and simulations are shown to be in agreement with the theoretical results.
\end{abstract}

\section{Introduction}

Cables/strings are flexible structural elements used in a wide span of engineering applications, such as cable towing operationsin marine applications. Depending on their physical properties and application areas, they exhibit vibration in the presence of disturbances. The most commonly used method for suppressing the cable vibration is to apply boundary kcontrollers. While a variety of boundary controllers have been proposed to control the motion of different cable/string systems, observer design for nonlinear cable/string systems has received little attention, which can be of great importance in the design of boundary controllers.

Boundary control of the cable/string systems have been investigated by several authors. Among others, Morgül, (1994) designed a boundary feedback controller for a system described by the wave equation where exponential stability of the closed loop is obtained for strictly proper transfer functions. Baicu et al. (1999) developed exponentially stabilizing controllers for the transverse vibration of a string-mass system modeled by one-dimensional wave equation. Shahruz \& Narasimha (1997) and Canbolat et al. (1998) presented exponentially stabilizing controllers for a onedimensional nonlinear string equation, allowing varying tension in the string. $\mathrm{Qu}$ (2000) devised a robust and adaptive controller to damp out the transverse oscillations of a stretched string, allowing nonlinear dynamics and their uncertainties in the model. The works mentioned above use a combination of the states at the boundary, namely the boundary slope, slope-rate and velocity, to design the stabilizing boundary control laws. Observer design based on Lyapunov analysis is well understood and widely used for linear systems. Recently, Demetriou (2001) presented the construction

*Centre for Ships and Ocean Structures. Norwegian University of Science and Technology, N-7491 Trondheim, Norway.

†Department of Engineering Cybernetics, Norwegian University of Science and Technology, N-7491 Trondheim, Norway. E-mail: yilmaz@itk.ntnu.no 
of natural observers for linear second order lumped and distributed parameter systems using parameter-dependent Lyapunov functions without resorting to a first order formulation. Unlike linear systems, construction of nonlinear observers lacked generality both from design and analysis point of view. Contraction theory is a recently developed nonlinear analysis tool which may be useful for solving a variety of nonlinear observer design problems. Lohmiller \& Slotine (1998) have presented Contraction theory through a series of publications. Kristiansen (2000) has used Contraction theory for the design of nonlinear observers.

In the paper, using Contraction theory, a nonlinear observer is designed for a general nonlinear cable/string FEM (Finite Element Method) model. The cable model is presented in the form of partial differential equations (PDE). Galerkin's method (Zienkiewicz \& Taylor, 2000) is then applied to obtain a set of ordinary differential equations such that the cable model is approximated by a FEM model. Based on the FEM model, a nonlinear observer is designed to estimate the cable configuration. It is shown that the estimated configuration converges exponentially to the actual configuration. Numerical results and simulations are shown to be in agreement with the theoretical results.

\section{Equations of Motion}

\subsection{Kinematics}

An inertial reference frame $i$ is defined with orthogonal unit vectors $\mathbf{i}, \mathbf{j}$ and $\mathbf{k}$ along the $x, y$ and $z$ axes, respectively. The spatial position of an arbitrary point on th center line of the initially stressed cable is given by the vector

$$
\mathbf{r}=x \mathbf{i}+y \mathbf{j}+z \mathbf{k}
$$

Consider an arbitrary point on the undeformed and initially stressed cable with the coordinates

$$
\mathbf{r}=x \mathbf{i}
$$

When the cable is deformed, the material point described by the material coordinates $\mathbf{r}$ will have the spatial coordinates

$$
\mathbf{r}=(x+\delta) \mathbf{i}+y \mathbf{j}+z \mathbf{k}
$$

as shown in Figure 1. This gives

$$
\left\|\frac{\partial \mathbf{r}}{\partial x}\right\| \approx 1+\frac{\partial \delta}{\partial x}+\frac{1}{2}\left(\frac{\partial y}{\partial x}\right)^{2}+\frac{1}{2}\left(\frac{\partial z}{\partial x}\right)^{2}
$$

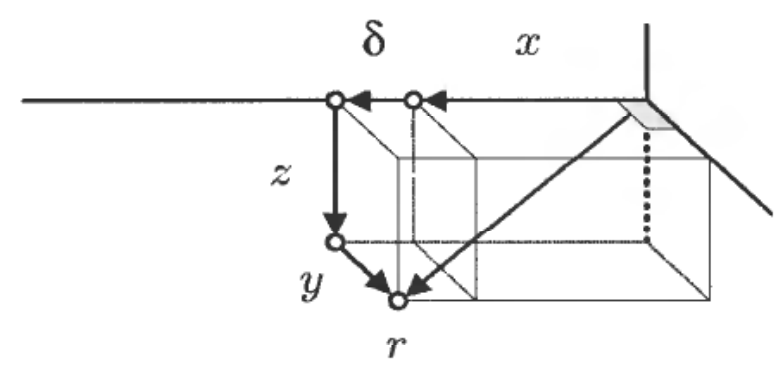

Figure 1. Kinematic consideration of cable/string. 
where right-hand side of equation (1) is the binomial approximation of $\left\|\frac{\partial \mathbf{r}}{\partial x}\right\|$. A cable frame $c$ of orthonormal vectors $\mathbf{t}$. $b$ and $\mathbf{n}$ is defined where $\mathbf{t}$ is the unit vector tangent to the cable such that

$$
\frac{\partial \mathbf{r}}{\partial x}=(1+\varepsilon) \mathbf{t}
$$

where $\varepsilon$ is the Lagrangian strain. Hence, from equations (1) and (2) the relation for the strain can be obtained as

$$
\varepsilon \approx \frac{\partial \delta}{\partial x}+\frac{1}{2}\left(\frac{\partial y}{\partial x}\right)^{2}+\frac{1}{2}\left(\frac{\partial z}{\partial x}\right)^{2}
$$

\subsection{Dynamics}

Consider a cable of length $L$. The dynamics of the cable is assumed to be determined by the tension in the cable and the inertial forces as shown in Figure 2. Using Hooke's law and equation (3), the tension $T$ in the cable can be expressed in the form

$$
\begin{aligned}
T(x) & =T_{0}+E_{A} \varepsilon \\
& =T_{0}+E_{A}\left[\frac{\partial \delta}{\partial x}+\frac{1}{2}\left(\frac{\partial y}{\partial x}\right)^{2}+\frac{1}{2}\left(\frac{\partial z}{\partial x}\right)^{2}\right]
\end{aligned}
$$

where $T_{0}$ is the constant tension in the initially stressed cable, $E_{\mathrm{A}}=E A, E$ is the Young's modulus and $A$ is the cross-section of the cable. Consider a material cable element of spatial length $d x$. Writing equilibrium of the forces in the $x, y$ and $z$ directions and using equation (4) gives the nonlinear coupled equations of motion

$$
\begin{aligned}
m \frac{\partial^{2} \delta}{\partial t^{2}} & =\frac{\partial T}{\partial x} \\
& =E_{A}\left(\frac{\partial^{2} \delta}{\partial x^{2}}+\frac{\partial y}{\partial x} \frac{\partial^{2} y}{\partial x^{2}}+\frac{\partial z}{\partial x} \frac{\partial^{2} z}{\partial x^{2}}\right)
\end{aligned}
$$

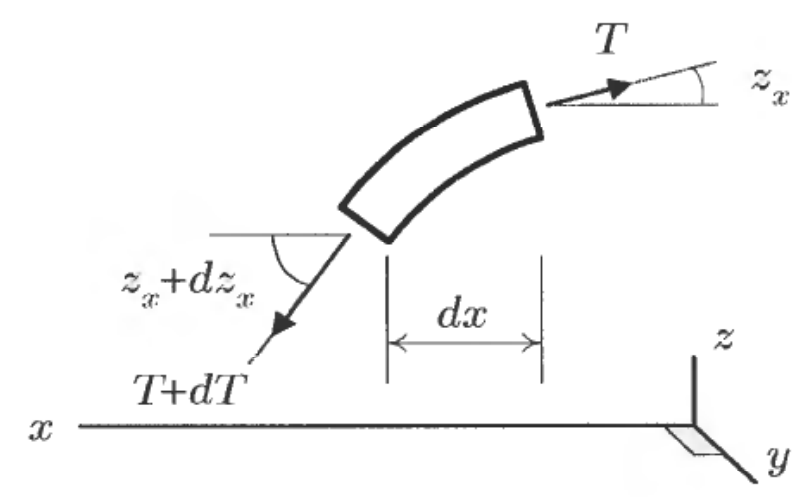

Figure 2. The forces acting on an elemental length $d x$. 


$$
\begin{aligned}
m \frac{\partial^{2} y}{\partial t^{2}} & =\frac{\partial}{\partial x}\left(T \frac{\partial y}{\partial x}\right) \\
& =E_{A}\left[\frac{\partial y}{\partial x} \frac{\partial^{2} \delta}{\partial x^{2}}+\left(\frac{\partial y}{\partial x}\right)^{2} \frac{\partial^{2} y}{\partial x^{2}}+\frac{\partial y}{\partial x} \frac{\partial z}{\partial x} \frac{\partial^{2} z}{\partial x^{2}}\right]+T \frac{\partial^{2} y}{\partial x^{2}} \\
m \frac{\partial^{2} z}{\partial t^{2}} & =\frac{\partial}{\partial x}\left(T \frac{\partial z}{\partial x}\right) \\
& =E_{A}\left[\frac{\partial z}{\partial x} \frac{\partial^{2} \delta}{\partial x^{2}}+\frac{\partial y}{\partial x} \frac{\partial z}{\partial x} \frac{\partial^{2} y}{\partial x^{2}}+\left(\frac{\partial z}{\partial x}\right)^{2} \frac{\partial^{2} z}{\partial x^{2}}\right]+T \frac{\partial^{2} z}{\partial x^{2}}
\end{aligned}
$$

for $x \in(0, L)$ and $t \geqslant 0$, where $m>0$ is the mass per unit length of the cable. Equations (5)-(7) can be put into matrix form

$$
\mathscr{H} \frac{\partial^{2} \mathbf{r}}{\partial t^{2}}=\mathscr{K} \frac{\partial^{2} \mathbf{r}}{\partial x^{2}}
$$

where

$$
\begin{aligned}
& \frac{\partial^{2} \mathbf{r}}{\partial t^{2}}=\left\{\frac{\partial^{2} \delta}{\partial t^{2}} \frac{\partial^{2} y}{\partial t^{2}} \frac{\partial^{2} z}{\partial t^{2}}\right\}^{T} \\
& \frac{\partial^{2} \mathbf{r}}{\partial x^{2}}=\left\{\frac{\partial^{2} \delta}{\partial x^{2}} \frac{\partial^{2} y}{\partial x^{2}} \frac{\partial^{2} z}{\partial x^{2}}\right\}^{T} \\
& \mathscr{K}=E_{A}\left[\begin{array}{ccc}
1 & \frac{\partial y}{\partial x} & \frac{\partial z}{\partial x} \\
\frac{\partial y}{\partial x} & \frac{T}{E_{A}}+\left(\frac{\partial y}{\partial x}\right)^{2} & \frac{\partial y}{\partial x} \frac{\partial z}{\partial x} \\
\frac{\partial z}{\partial x} & \frac{\partial y}{\partial x} \frac{\partial z}{\partial x} & \frac{T}{E_{A}}+\left(\frac{\partial z}{\partial x}\right)^{2}
\end{array}\right]
\end{aligned}
$$

and $. \mathscr{M}=m \mathbf{I}_{m}, \mathbf{I}_{m}$ is the $3 \times 3$ idenity matrix. The initial conditions for equation (8) are given by

$$
\begin{array}{r}
\mathbf{r}(x, 0)=\mathbf{b}_{1}(x) \\
\partial \mathbf{r}(x, 0) / \partial t=\mathbf{b}_{2}(x)
\end{array}
$$

where $\mathbf{b}_{1}(x)$ and $\mathbf{b}_{2}(x)$ are assumed to be smooth functions. The boundary conditions are given by

$$
\begin{gathered}
\mathbf{r}(0, t)=\mathbf{0} \\
T(L) \partial \mathbf{r}(L, t) / \partial x=\mathbf{u}(t)
\end{gathered}
$$

where the function $\mathbf{u}(t)$ is the boundary control input. 


\subsection{FEM Model}

In this section, the cable/string model presented in the form of PDEs in equation (8) will be approximated by a FEM model to obtain a set of ordinary differential equations. Without loss of generality and for the sake of simplicity in the derivations and analysis which will follow later in this paper, the motion of the cable is confined in $x y$-plane. Ignoring $\delta$ and $z$ components in equation (8) and choosing the parameters $m, T_{0}$ and $E_{A}$ as unity renders the following nonlinear scalar equation of motion for the cable/string (Shahruz \& Narasimha, 1997; Qu, 2000)

$$
\frac{\partial^{2} y}{\partial t^{2}}=\left[1+\frac{3}{2}\left(\frac{\partial y}{\partial x}\right)^{2}\right] \frac{\partial^{2} y}{\partial x^{2}}
$$

The initial conditions for equation (9) are given by

$$
\begin{array}{r}
y(x, 0)=b_{1}(x) \\
\partial y(x, 0) / \partial t=b_{2}(x)
\end{array}
$$

where $b_{1}(x)$ and $b_{2}(x)$ are assumed to be smooth functions of $x$. The boundary conditions are given by

$$
\begin{gathered}
y(0, t)=0 \\
T(L, t) \partial y(L, t) / \partial x=u(t)
\end{gathered}
$$

where $u(t)$ is the boundary control input. To obtain a set of ordinary differential equations, Galerkin's method is applied to equation (9). The cable is divided into $n$ elements, where nodal points are enumerated from 0 to $n$. Let $h=L / n$ be the length of each cable segment. For $x \in[0, L]$, Galerkin's method yields the following discretized FEM model

$$
\mathbf{M y}+\mathbf{k}(\mathbf{y})=\mathbf{u}(t)
$$

where

$$
\mathbf{y}=\left\{\begin{array}{c}
y_{1} \\
y_{2} \\
\vdots \\
y_{n}
\end{array}\right\}, \quad \boldsymbol{\kappa}(\mathbf{y})=\left\{\begin{array}{c}
k_{1}(\mathbf{y}) \\
k_{2}(\mathbf{y}) \\
\vdots \\
k_{n}(\mathbf{y})
\end{array}\right\}, \quad \mathbf{u}(t)=\left\{\begin{array}{c}
0 \\
0 \\
\vdots \\
u(t)
\end{array}\right\}
$$

$$
\mathbf{M}=\frac{h}{6}\left[\begin{array}{ccccc}
4 & 1 & 0 & & \\
1 & 4 & \ddots & \ddots & \\
0 & \ddots & \ddots & & 0 \\
& \ddots & & 4 & 1 \\
& & 0 & 1 & 2
\end{array}\right]
$$

and

$$
k_{1}(\mathbf{y})=\frac{1}{h}\left(2 y_{1}-y_{2}\right)+\frac{1}{2 h^{3}} y_{1}^{3}+\frac{1}{2 h^{3}}\left(y_{2}-y_{1}\right)^{2} y_{1}-\frac{1}{2 h^{3}}\left(y_{2}-y_{1}\right)^{2} y_{2}
$$




$$
\begin{aligned}
k_{j}(\mathbf{y})= & \frac{1}{h}\left(-y_{j-1}+2 y_{j}-y_{j+1}\right)-\frac{1}{2 h^{3}}\left(y_{j}-y_{j-1}\right)^{2} y_{j-1}+\frac{1}{2 h^{3}}\left[\left(y_{j}-y_{j-1}\right)^{2} y_{j}\right. \\
& \left.+\left(y_{j+1}-y_{j}\right)^{2} y_{j}\right]-\frac{1}{2 h^{3}}\left(y_{j+1}-y_{j}\right)^{2} y_{j+1} \\
& \vdots \\
k_{n}(\mathbf{y})= & \frac{1}{h}\left(y_{n}-y_{n-1}\right)-\frac{1}{2 h^{3}}\left(y_{n}-y_{n-1}\right)^{2} y_{n-1}+\frac{1}{2 h^{3}}\left(y_{n}-y_{n-1}\right)^{2} y_{n}
\end{aligned}
$$

for $j=2, \ldots, n-1$.

\section{Observer Design}

In this section, a nonlinear observer will be designed for the system given in equation (10) using Contraction theory (Jouffroy et al., 2004 and Lohmiller \& Slotine, 1998).

\subsection{Observer dynamics}

The observer structure copies the second-order nonlinear model dynamics in equation (10) with a correction term, and is given by

$$
\mathbf{M} \ddot{\mathbf{y}}+\boldsymbol{\kappa} \hat{\mathbf{y}})=\mathbf{u}(t)+\mathbf{H}\{\dot{\mathbf{y}}-\hat{\mathbf{y}}\}
$$

Here, $\mathbf{H}$ is the observer gain matrix, having the form

$$
\mathbf{H}=\operatorname{diag}\left(0, \ldots, 0, K_{v}\right), \quad K_{v}>0
$$

where we assume that the only measurement available is the velocity $\dot{y}_{n}(L)$ at the boundary, $x=L$. Computing the virtual dynamics of equation (14) gives

$$
\mathbf{M} \delta \ddot{\mathbf{y}}+\frac{\partial \mathbf{\kappa}}{\partial \hat{\mathbf{y}}}(\hat{\mathbf{y}}) \delta \hat{\mathbf{y}}=-\mathbf{H} \delta \hat{\mathbf{y}}
$$

where the Jacobian matrix is given by

$$
\frac{\partial \mathbf{k}}{\partial \hat{\mathbf{y}}}(\hat{\mathbf{y}})=\left[\begin{array}{ccccc}
\kappa_{11} & \kappa_{12} & 0 & & \\
\kappa_{21} & \kappa_{22} & \kappa_{23} & \ddots & \\
0 & \kappa_{32} & \ddots & \ddots & 0 \\
& \ddots & \ddots & \kappa_{n-1, n-1} & \kappa_{n-1, n} \\
& & 0 & \kappa_{n, n-1} & \kappa_{n, n}
\end{array}\right]
$$

with the entries

$$
\begin{aligned}
& \kappa_{11}=\frac{2}{h}+\frac{3}{2 h^{3}}\left[\begin{array}{ll}
\hat{y}_{1}^{2}+\left(\hat{y}_{2}\right. & \left.\hat{y}_{1}\right)^{2}
\end{array}\right] \\
& \kappa_{12}=-\frac{1}{h}-\frac{3}{2 h^{3}}\left(\hat{y}_{2}-\hat{y}_{1}\right)^{2}
\end{aligned}
$$




$$
\begin{aligned}
\kappa_{j(j-1)}= & -\frac{1}{h}-\frac{3}{2 h^{3}}\left(\hat{y}_{j}-\hat{y}_{j-1}\right)^{2} \\
\kappa_{j j}= & \frac{2}{h}+\frac{3}{2 h^{3}}\left[\left(\hat{y}_{j}-\hat{y}_{j-1}\right)^{2}+\left(\hat{y}_{j}-\hat{y}_{j+1}\right)^{2}\right] \\
\kappa_{j(j+1)}= & -\frac{1}{h}-\frac{3}{2 h^{3}}\left(\hat{y}_{j}-\hat{y}_{j+1}\right)^{2} \\
& \vdots \\
\kappa_{n(n-1)}= & -\frac{1}{h}-\frac{3}{2 h^{3}}\left(\hat{y}_{n}-\hat{y}_{n-1}\right)^{2} \\
\kappa_{n n}= & \frac{1}{h}+\frac{3}{2 h^{3}}\left(\hat{y}_{n}-\hat{y}_{n-1}\right)^{2}
\end{aligned}
$$

for $j=2, \ldots, n-1$. Note that each $\kappa$ term is a quadratic scalar function, rendering off-diagonal terms negative definite and diagonal terms positive definite for every $\hat{\mathbf{y}}$. Let

$$
\mathbf{K}=\frac{\partial \mathbf{K}}{\partial \hat{\mathbf{y}}}(\hat{\mathbf{y}})
$$

where $\mathbf{K}$ is the $n \times n$ matrix. Hence, the virtual dynamics in equation (16) can be rewritten in first order form

$$
\frac{d}{d t}\left\{\begin{array}{l}
\delta \hat{\mathbf{y}} \\
\delta \hat{\mathbf{y}}
\end{array}\right\}=\mathbf{A}\left\{\begin{array}{l}
\delta \hat{\mathbf{y}} \\
\delta \hat{\mathbf{y}}
\end{array}\right\}
$$

where

$$
\begin{aligned}
& \mathbf{A}=\left[\begin{array}{cc}
\mathbf{0} & \mathbf{I} \\
-\mathbf{E} & -\mathbf{F}
\end{array}\right] \\
& \mathbf{E}=\mathbf{M}^{-1} \mathbf{K}, \quad \mathbf{F}=\mathbf{M}^{-1} \mathbf{H}
\end{aligned}
$$

and $\mathbf{0}$ is the $n \times n$ zero matrix and $\mathbf{I}$ is the $n \times n$ identity matrix.

\subsection{Stability properties of the observer}

To establish contraction, it can be shown that all eigenvalues of the system matrix A satisfy $\operatorname{Re} \lambda_{i}<0$. This is established as follows: Consider equation (18) and the eigenvalue problem

$$
\mathbf{A}\left\{\begin{array}{l}
\mathbf{x}_{1} \\
\mathbf{x}_{2}
\end{array}\right\}=\lambda\left\{\begin{array}{l}
\mathbf{x}_{1} \\
\mathbf{x}_{2}
\end{array}\right\} \Leftrightarrow\left\{\begin{array}{l}
\lambda \mathbf{x}_{1}=\mathbf{x}_{2} \\
\lambda \mathbf{x}_{2}=-\mathbf{E} \mathbf{x}_{1}-\mathbf{F} \mathbf{x}_{2}
\end{array}\right.
$$


1. Assume that $\lambda \geqslant 0$ and real. From equation (19) we have

$$
\begin{aligned}
\lambda \mathbf{x}_{1} & =\mathbf{x}_{2} \\
-\lambda^{2} \mathbf{x}_{1} & =(\mathbf{E}+\lambda \mathbf{F}) \mathbf{x}_{1}
\end{aligned}
$$

where $\mathbf{E}$ is positive definite and $\mathbf{F}$ is positive semi-definite. But since $(\mathbf{E}+\lambda \mathbf{F})$ is positive definite, the assumption is false by contradiction.

2. Assume that $\lambda$ is not real. From equation (20) we can write

$$
\lambda^{2} \mathbf{x}_{1}^{T} \mathbf{x}_{1}+\lambda \mathbf{x}_{1}^{T} \mathbf{F} \mathbf{x}_{1}+\mathbf{x}_{1}^{T} \mathbf{E} \mathbf{x}_{1}=0
$$

Selecting $\left|\mathbf{x}_{1}\right|=1$ gives

$$
\lambda^{2}+\lambda \mathbf{x}_{1}^{T} \mathbf{F} \mathbf{x}_{1}+\mathbf{x}_{1}^{T} \mathbf{E} \mathbf{x}_{1}=0
$$

The characteristic equation in (21) has the roots

$$
\lambda=\frac{1}{2}\left\{-\mathbf{x}_{1}^{T} \mathbf{F} \mathbf{x}_{1} \pm\left[\left(\mathbf{x}_{1}^{T} \mathbf{F} \mathbf{x}_{1}\right)^{2}-4 \mathbf{x}_{1}^{T} \mathbf{E} \mathbf{x}_{1}\right]^{1 / 2}\right\}
$$

which implies that

$$
\operatorname{Re} \lambda=-\frac{1}{2} \mathbf{x}_{1}^{T} \mathbf{F} \mathbf{x}_{1} \leqslant 0
$$

as $\lambda$ is not real by assumption. If $\operatorname{Re} \lambda=0$ then $\mathbf{F x}_{1}=0$ and

$$
\mathbf{F x}_{1}=0 \Leftrightarrow \mathbf{x}_{1}=\left\{\begin{array}{c}
x_{1} \\
\vdots \\
x_{n-1} \\
0
\end{array}\right\}
$$

where

$$
\mathbf{F}=\left[\begin{array}{cccc}
0 & \cdots & 0 & * \\
& \ddots & \vdots & \vdots \\
& & 0 & * \\
& & & K_{v}
\end{array}\right\}
$$

but from equation (20) we can conclude that such a vector $\mathbf{x}_{1}$ cannot be an eigenvector of the positive definite matrix $(\mathbf{E}+\lambda \mathbf{F})$. Hence, all eigenvalues of A satisfy $\operatorname{Re} \lambda_{i}<0$.

Since $\mathbf{A}$ is a stability matrix with $\operatorname{Re} \lambda_{i}<0$, there exists a uniformly positive definite metric $\boldsymbol{\Gamma}$ under which the observer is contracting, i.e. that the virtual displacement

$$
\delta \mathbf{z}=\left\{\begin{array}{l}
\delta \hat{\mathbf{y}} \\
\delta \hat{\mathbf{y}}
\end{array}\right\}
$$

converges exponentially to zero as shown in Figure 3. Furthermore, there exist two strictly positive numbers $\alpha$ and $\gamma$ such that for any two trajectories starting from 


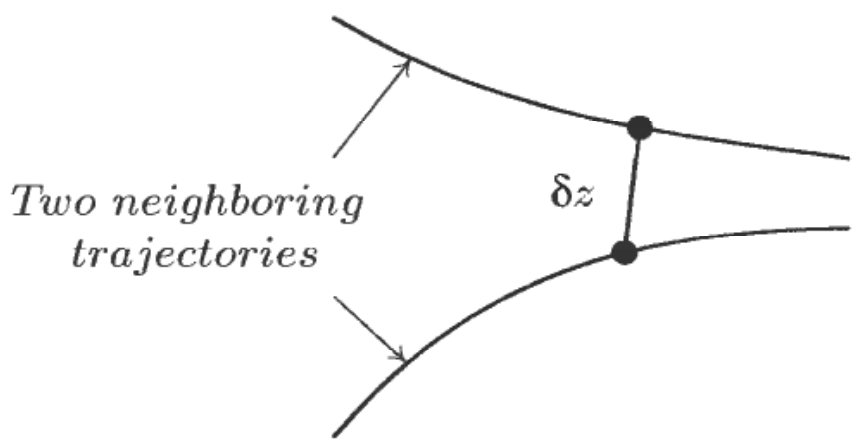

Figure 3. The virtual displacement $\delta \mathbf{z}$.

$\mathbf{z}\left(t=t_{0}\right)$ and $\hat{\mathbf{z}}\left(t=t_{0}\right)$, the observer error dynamics is exponentially stable in the sense that

$$
\|\mathbf{z}(t)-\hat{\mathbf{z}}(t)\| \leqslant \alpha\left\|\mathbf{z}\left(t_{0}\right)-\ddot{\mathbf{z}}\left(t_{0}\right)\right\| e^{-r\left(t-t_{0}\right)}
$$

for $t \geqslant t_{0}$ everywhere in the region in which equation (17) holds (Jouffroy et al., 2004).

\section{Numerical Results and Simulation}

The observer proposed in equation (14) is solved numerically. The results of the simulation are shown in Figures 4-11. In the simulations, a cable of unit length is discretized with $n=10$, where $n=10$ is the node at the boundary and $n=0$ is the stationary end point. The observer gain is chosen as $K_{v}=1$. During the simulations,

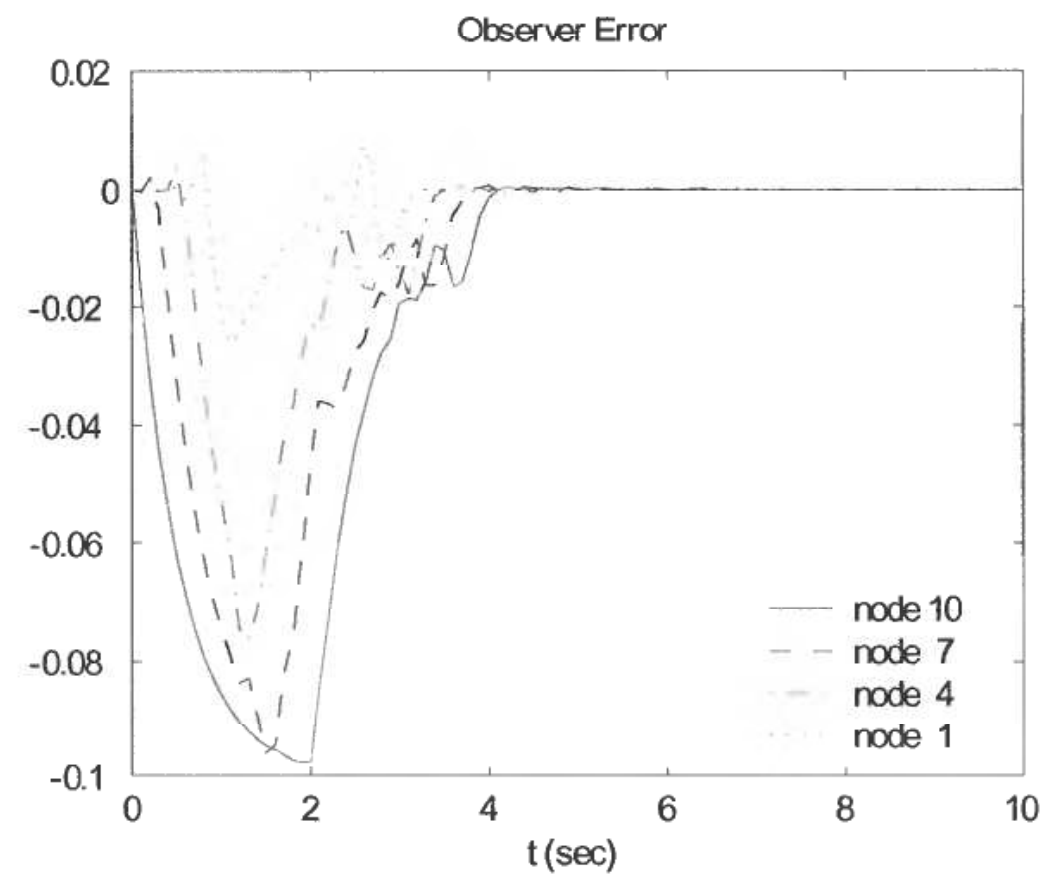

Figure 4. The boundary node follows a step reference. The observer was switched on at time $t=2 \mathrm{sec}$. 
Cable configuration in $x y$-plane

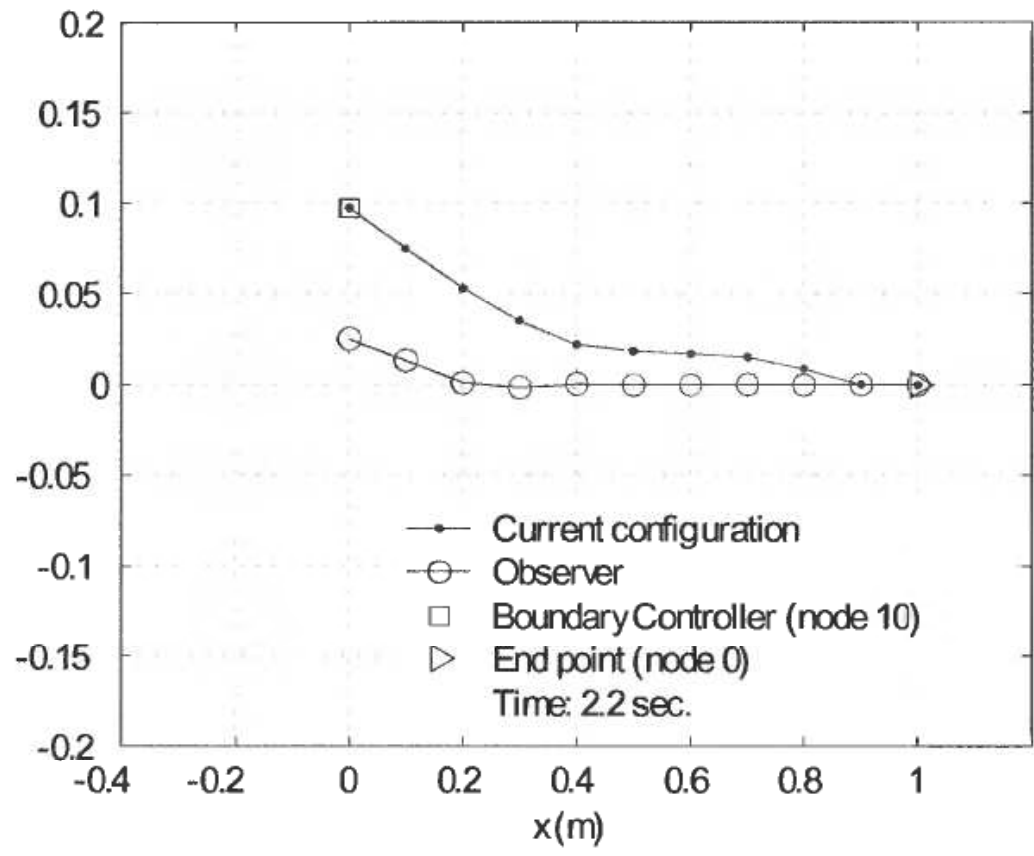

Figure 5. Configurations at $t=2.2 \mathrm{sec}$.

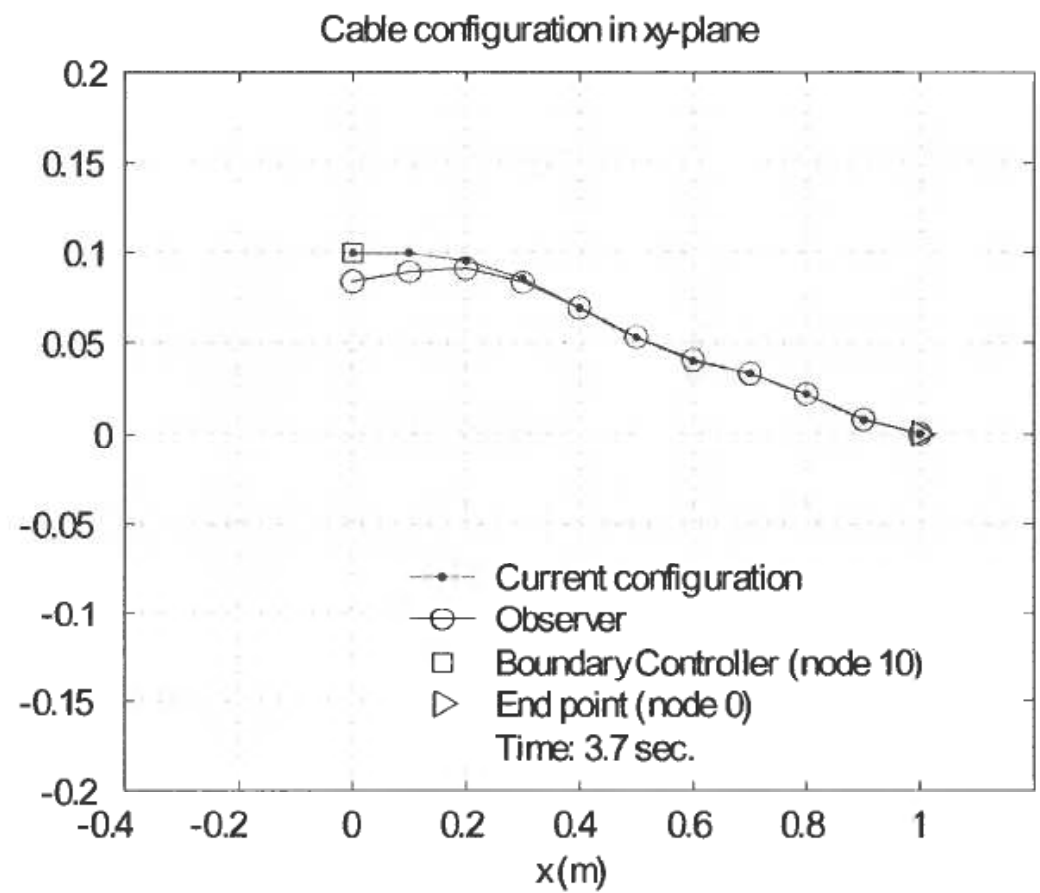

Figure 6. Configurations at $t=3.7 \mathrm{sec}$. 


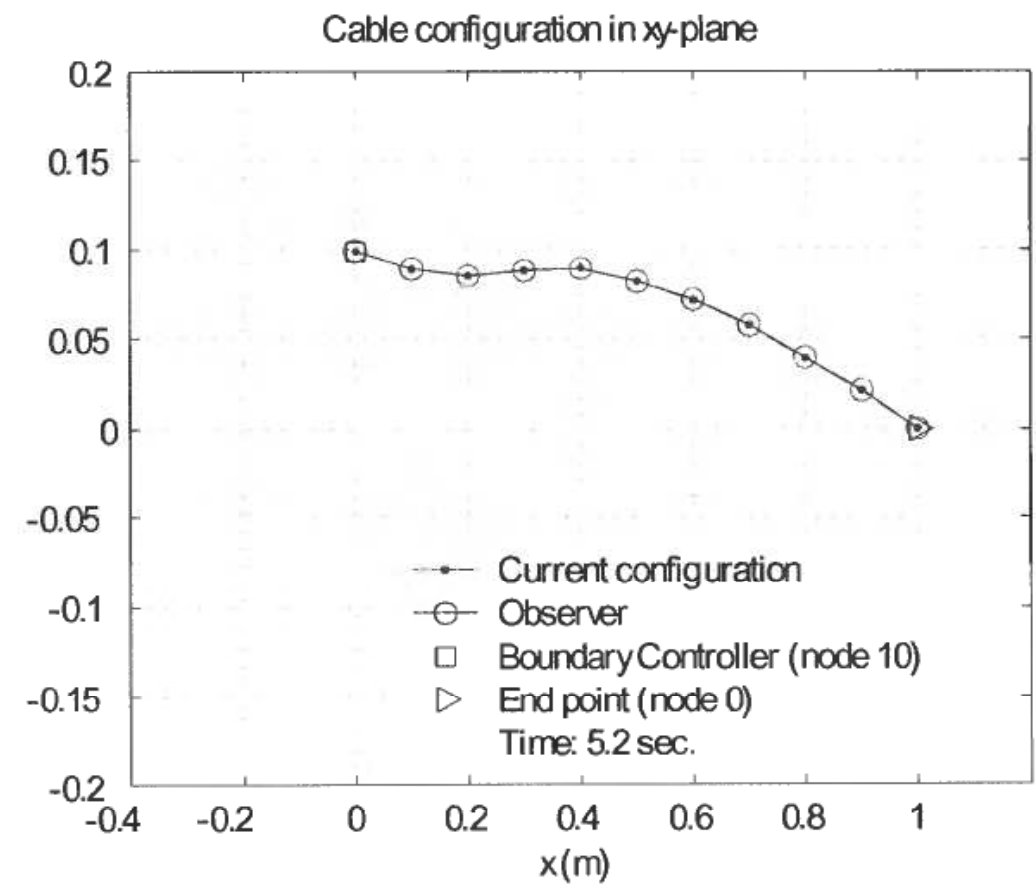

Figure 7. Configurations at $t=5.2 \mathrm{sec}$.

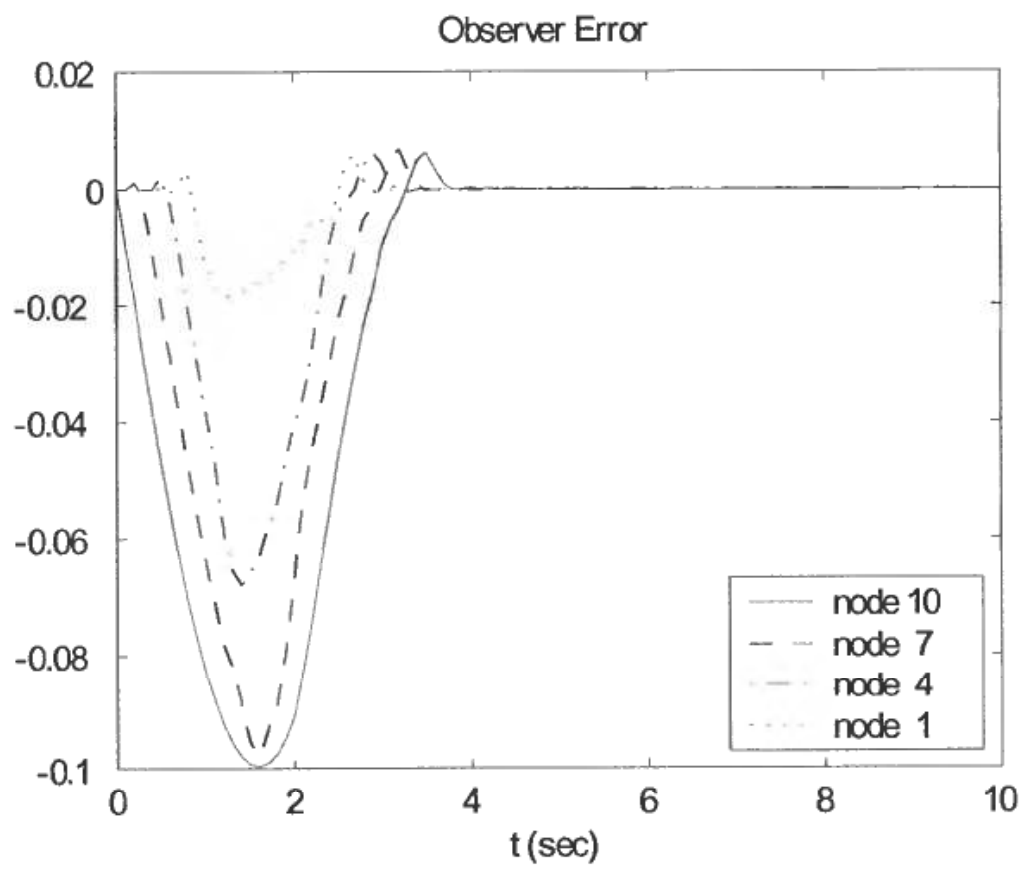

Figure 8. The boundary node follows a varying reference. The observer was switched on at $t=2$ sec. 


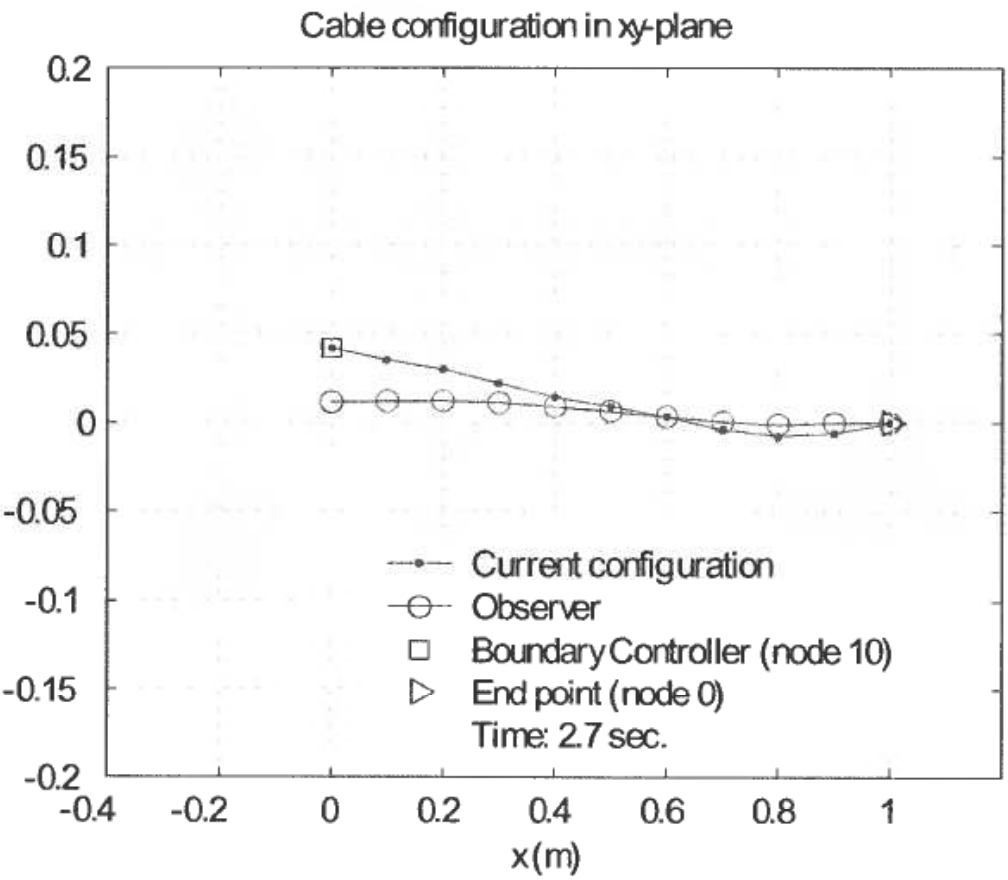

Figure 9. Configurations at $t=2.7 \mathrm{sec}$.

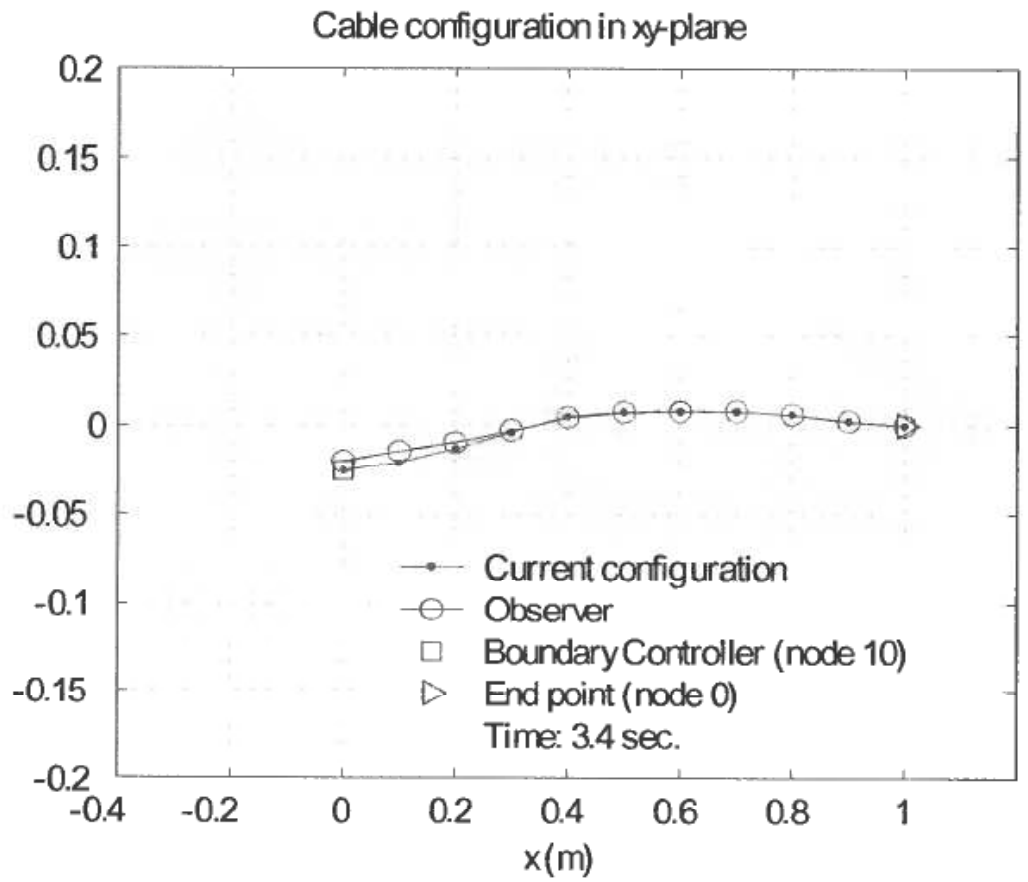

Figure 10. Configurations at $t=3.4 \mathrm{sec}$. 


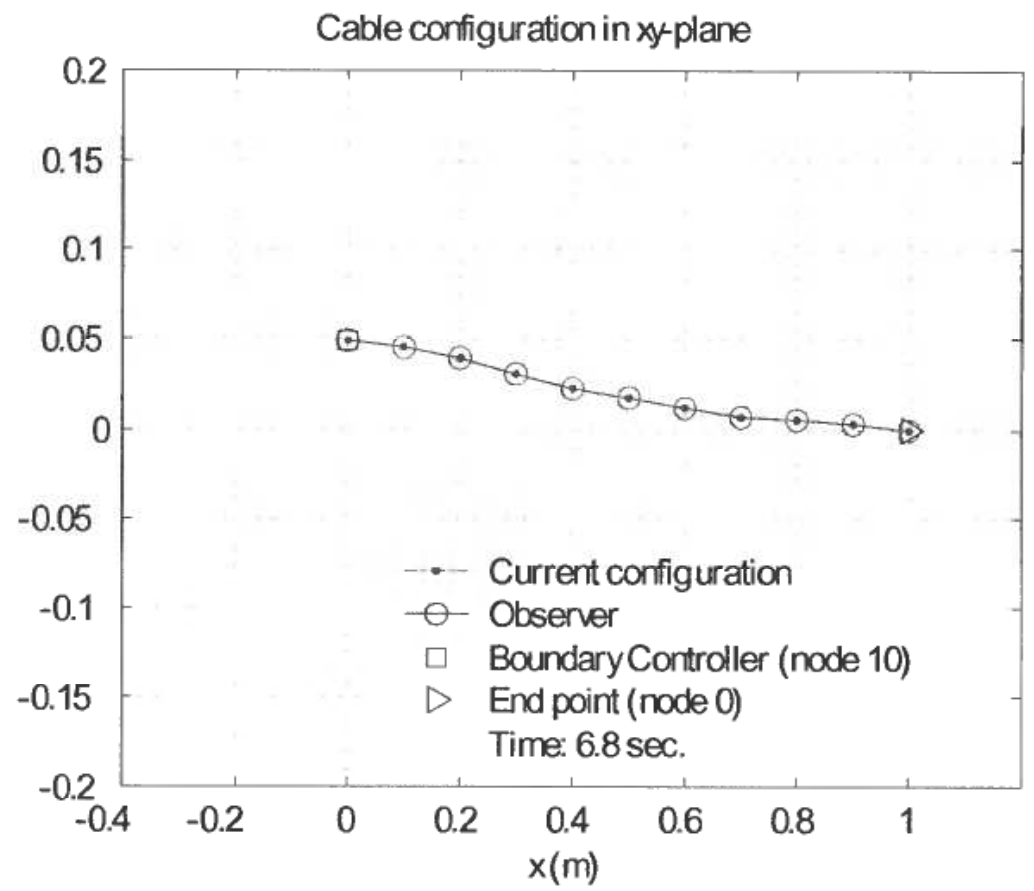

Figure 11. Configurations at $t=6.8 \mathrm{sec}$.

two different motions of the boundary node are considered. In both cases, the observer is switched on at $t=2 \mathrm{sec}$. First, the boundary node is assigned to follow a step reference. Figure 4 shows the observer error at the nodes $n=1,4,7$ and 10 when the boundary node follows a step reference whereas Figures 5-7 are the snapshots taken during the simulation which show the current and estimated configurations at times $t=2.2,3.7$ and $5.2 \mathrm{sec}$. Similarly, the boundary node is then assigned to follow a time-varying reference whereas Figures 9-11 show the current and estimated configurations at times $t=2.7,3.4$ and $6.8 \mathrm{sec}$. As seen in Figure 4 and Figure 8, the observer error starts decreasing rapidly and the estimated configuration converges to the actual configuration which demonstrates the contracting property of the observer dynamics. The results from the simulations are thus in agreement with the theory presented in this paper.

\section{Conclusions}

Contraction theory is a recently developed nonlinear analysis tool which may be useful for solving a variety of nonlinear control problems. In this paper, using Contraction theory, a nonlinear observer has been designed for a general nonlinear cable/string FEM model. The cable model has been presented in the form of PDFs. Galerkin's method has been then applied to obtain a set of ordinary differential equations such that the cable model has been approximated by a FEM model. Based on the FEM model, a nonlinear observer was designed to estimate the cable configuration. It is shown that the estimated configuration converges exponentially to the actual configuration. Numerical results and simulations have been shown to be in agreement with the theoretical results. 


\section{Acknowledgement}

This work was funded by the Norwegian Research Council under the Strategic University Program in Marine Cybernetics. The authors would like to thank Dr J. Jouffroy for the interesting discussions on Contraction theory.

\section{References}

Baicu, C. F, Rahn, C. D. \& Dawson, D. M. (1999). Exponentially stabilizing boundary control of string-mass systems, Journal of Vibration and Control, 5, pp. 491-502.

Canbolat, H., Dawson, D., Nagarkatti, S. P. \& Costic, B. (1998). Boundary control for a general class of string models, Proceedings of the American Control Conference. Pennsylvania.

Demetriou, M. A. (2001). Natural observers for second order lumped and distributed parameter systems using parameter-dependent lyapunov functions, Proceedings of the American Control Conference, Arlington, VA.

JoUfFroY, J., FoSSEN. T. I. \& SLOTINE, J.-J. E. (2004). Methodological remarks on contraction theory, exponentially stability and lyapunov functions. Submitted to Automatica.

KristiansEN, D. (2000). Modeling of Cylinder Gyroscopes and Observer Design for Nonlinear Oscillatons. PhD thesis. Dept. of Engineering Cybernetics, Norwegian Univ. of Sci. and Tech.

Lohmiller, W. \& Slotine, J.-J. E. (1998). On contraction analysis for non-linear systems. Automatica, 34(6), pp. 683-696.

MORGÜL, Ö. (1994). A dynamic control law for the wave equation, Automatica, 30, pp. $1785-1792$.

Qu, Z. (2000). Robust and adaptive boundary control of a stretched string, Proceedings of the American Control Conference, Illinois.

ShahruZ, S. M. \& Narasimha, C. A. (1997). Suppression of vibration in stretched strings by the boundary control, Journal of Sound and Vibration, 204, pp. 835-840.

Zienkiewicz, O. C. \& TAYlor, R. L. (2000). The Finite Element Method, 5th edn, ButterworthHeinemann, Oxford, UK. 